Ann. Zootech., I968, 17 (4), 467-468.

\title{
HÉRITABILITÉ DES CARACTÈRES DE PONTE SUIVANT LA DATE D'ÉCLOSION
}

\author{
P. MÉRAT \\ Station centrale de Génétique animale \\ Centre national de Recherches zootechniques, 78 -Jouy-en-Josas \\ Institut national de la Recherche agronomique
}

Nous avions signalé (MÉRaT, 1956) que, sur 7 générations appartenant à deux troupeaux de volailles, nés au printemps et élevés au sol en lumière naturelle, l'héritabilité de l'âge au premier œuf, calculée à partir des variances entre sœurs et demi-sours, différait suivant qu'on l'estimait sur les poulettes écloses au début ou à la fin de la saison pedigree : composante "mère "plus grande pour les premières, composante " père " plus importante à proportion pour les dernières.

Des données complémentaires recueillies depuis confirment cette tendance. Elles portent sur six générations successives (I954 à 1959) d'une souche "synthétique " dont les éclosions pedigree avaient lieu en mars-avril de chaque année. Le nombre de semaines consécutives d'éclosion était de 5 ou 6 . Tous les lots descendaient des mêmes parents; il y avait, par année, de $I_{3}$ à 20 pères, une centaine de mères et de 200 à 400 filles pour chaque groupe.

Le tableau $\mathrm{I}$ indique, pour chaque génération, les valeurs de l'héritabilité estimée d'après la composante "père " et "mère " de la variance, respectivement pour les trois premiers et les 3 (ou 2) derniers lots éclos.

TABIEAU I

Héritabilités estimées à partir de la composante "père $\left(h_{\mathrm{s}}\right)$ et "mère " $\left(h_{\mathrm{D}}^{2}\right)$ de la variance, pour l'áge au premier auf des poulettes écloses en début et en fin de saison

\begin{tabular}{|c|c|c|c|c|}
\hline \multirow[b]{2}{*}{ Année } & \multicolumn{2}{|c|}{ Premiers lots } & \multicolumn{2}{|c|}{ Derniers lots } \\
\hline & $\begin{array}{c}h^{2} \mathbf{D} \\
\text { (d'après } \\
\text { la composante } \\
\text { "mère ") }\end{array}$ & $\begin{array}{c}h^{2} \mathrm{~s} \\
\text { (d'après } \\
\text { la composante } \\
\text { "père ") }\end{array}$ & $h^{2} \mathrm{n}$ & $h^{2} \mathrm{~s}$ \\
\hline $195^{\prime}$ & 0,70 & 0,27 & 0,75 & 0,57 \\
\hline 1955 & 0,31 & 0,59 & 0,21 & 0,22 \\
\hline 1956 & 0,55 & 0,12 & 0,30 & 0,19 \\
\hline 1957 & 0,47 & 0,21 & 0,18 & 0,29 \\
\hline 1958 & 1,18 & 0,13 & 1,00 & 0,14 \\
\hline 1959 & 0,55 & 0,31 & $(-0,36)$ & 0,29 \\
\hline Moyenne non pondérée & 0,63 & 0,27 & 0,35 & 0,28 \\
\hline
\end{tabular}


La tendance est la même que celle relatée dans notre première publication : héritabilité estimée d'après la "composante mère "plus grande pour les premiers lots (5 fois sur 6) ; héritabilité "père " plus élevée - au moins proportionnellement - dans les derniers lots.

En réunissant ces données avec nos résultats antérieurs, on constate, ro fois sur $\mathbf{I}_{3}$ au total, un écart de même sens pour l'héritabilité "mère ", la différence entre premiers et demiers lots pour ce paramètre étant très faible dans les trois autres cas.

La variance phénotypicue est plus grande pour les lots nés plus tardivement, et il en est de même de la variance intra-familles. On peut penser que cela correspond a une augmentation de la variance "environnement".

Quant à la valeur plus grande de la "composante mère " dans les premiers lots, elle peut être due à une variance génétique (additive ou non) plus importante en valeur relative, ou à des effets maternels non manifestés dans les lots tardifs.

Une comparaison analogue de l'héritabilité du nombre d'ceufs pondus entre l'entrée en ponte et le 3 I décembre, pour les animaux nés tôt ou tard, ne montre pas de tendance comparable évidente ; pour le poids fin décembre, mesuré les trois dernières années, l'héritabilité ( $h_{\mathrm{n}}^{2}$ aussi bien que $h_{\mathrm{S}}^{2}$ ) apparaît plus élevée pour les lots tardifs.

Le fait que l'expression du génotype pour les caractères " ponte " diffère suivant la date de naissance, est suggéré encore par l'existence, pour les premiers lots, d'une corrélation phénotypique légère, mais significative, entre âge au premier couf et poids à 8 semaines, cette corrélation étant pratiquement nulle dans les demiers lots.

Cependant, cela ne paritit pas se refléter dans l'interaction familles $\times$ lot, non significative sur l'ensemble de ces données, tant pour l'âge au premier ouf que pour la ponte d'automne et d'hiver (données non publiées) ( ${ }^{1}$.

Rę̧u pour publication en septembre 1968.

\author{
SUMMARY \\ HERITABILITY OF AGE AT FIRST EGG DEPENDING \\ UPON DATE OF HATCII
}

In six successive generations of a "synthetic " strain, hatched in spring and housed on floor without artificial lighting (1954 to 1959), the heritability of age at first egg, evaluated from the partition of variance between and within full and half-sister families, was different for pullets hatched at the beginning or at the end of the breeding season : the " dam " component was higher for the first ones, the " sire " component had a greater proportional importance for the last ones.

These data further support our previous results.

\title{
RÉFÉRENCE BIBLIOGRAPHIQUE
}

MÉRat P., I956. L'effet de la date d'éclosion sur le résultat de la sélection pour la précocité sexuelle des volailles. Ann. Zootech., 5, i 73-194.

(1) La variance intra-famille dépendant du lot, le test d'interaction famille $\times$ lot pourrait être mis en question, mais la différence de variance ne paraît pas assez considérable pour changer le sens de nos conclusions. 